\title{
Clinical importance of glutathione-s-transferase enzyme polymorphisms in Cancer
}

\begin{abstract}
Glutathione S-transferase (GST) enzyme levels, which is the phase II enzyme of biotransformation enzyme group, responsible for detoxification of xenobiotics, are associated with the risk of predisposition to various types of cancer as well as diseases such as asthma, diabetes, Parkinson's. In this review, we attempt to briefly summarize the association with the polymorphisms of GSTM1, GSTT1, GSTP, and specific cancer types.
\end{abstract}

Volume 3 Issue 6 - 2018

\author{
Zehra Okat \\ Üsküdar Zeynep Kamil Vocational and Technical Anatolian Highs \\ School,Turkey

\begin{abstract}
Correspondence: Zehra Okat, Üsküdar Zeynep Kamil Vocational and Technical Anatolian High School, Istanbul. Arakiyeci Cafer Mah. Nuh Kuyusu Cad. No 4I Üsküdar/İstanbul, Tel 05066109846, Email zehraoket1980@gmail.com
\end{abstract}

Received: October 23, 2018 | Published: November 19, 2018

\section{Introduction}

GSTs, which are the members of the antioxidant enzyme group, are also regulated by oxidant stress. The GST gene family in the dimeric enzyme structure known to be expressed in various tissue. GSTclass enzymes, by means of the reduced level of glutathione (GSH), conjugate various reactivate electrophiles. And also responsible for an essential function in cellular defense by reducing their levels. Genetic polymorphisms in genes encoding certain GST-class enzymes, such as homozygous deletions in GSTM1, and GSTT1 genes, result in wholly loss of GSTM1 or GSTT1 protein products. ${ }^{1}$ Through previous metaanalysis studies, there is evidence that the null GSTT1 genotype may be related with the increased risk of different cancers such as breast cancer, lung cancer, stomach cancer, and liver cancer., ${ }^{2,3}$

\section{Cancer types}

\section{Bladder cancer}

In the pathogenesis of this cancer type; a wide range of aromatic, and heterocyclic amine sequences present in the chemical, and transport industry, present in the diet, and cigarette smoke, are considered to be carcinogenic to the bladder. Accordingly, exposure to arylamines is expected to ascend the risk of bladder cancer by 40-100 times. ${ }^{4}$ Upregulation of different types of GST in bladder transitional cell carcinoma (TCC) is thought to affect TCC growth by causing inhibition of apoptosis. ${ }^{5}$ A study in South Korea found a strong link between GSTM1 null genotype, and susceptibility to bladder cancer. ${ }^{6}$ In an analysis conducted in the Korean population; in patients with a history of tuberculosis, and bronchial asthma, rapid acetylator genotypes, and a combination of GSTM1 null or GSTT1 null genotypes, it was found to be an an increased risk for bladder cancer. $^{7}$

\section{Breast cancer}

Breast cancer etiology remains to be unclear. However, many different risk factors have been reported, including an increased systemic concentration of reactive oxygen species. ${ }^{8}$ It is envisaged that free reactive species may induce tumor onset in this case where they can cause irreversible damage such as point mutations in DNA or chromosomal aberration. For these reasons, it is believed that biological agents that may be involved in reducing levels of reactive species may act as a key role in reducing the progression of developing breast cancer. ${ }^{8}$ In terms of susceptibility to breast cancer, low-sensitivity susceptibility-related gene groups, the role of GST gene in the detoxification mechanism can be shown first. ${ }^{9}$ The gene family of GSTs represents one of the enzymes of phase II drug metabolism, which is a very important function in detoxifying many carcinogens, and reactive metabolite intermediates, potentially causing damage to cells, and causing cancers, including breast. ${ }^{10}$ In the case-control analyzes performed, a positive association between GSTT1 null, and breast cancer risk was shown. ${ }^{11}$ It was determined that homozygous deletions of GSTT1, and GSTM1 genes, and breast cancer development were an increased. ${ }^{12}$ Beside these studies; in a study conducted in the South Indian population, GSTM1 homozygous null genotype has been demonstrated not to perform a function in breast cancer risk. ${ }^{13}$

\section{Colorectal cancer}

External environmental factors may play a significant responsibility in the development of colorectal cancer. ${ }^{14}$ Accordingly, it is thought that the effective metabolism of environmental carcinogens such as polycyclic aromatic hydrocarbons (PAHs), heterocyclic amines, and nitrosamines may play a protective role against colorectal cancer. ${ }^{15}$ The ability of individuals to detoxify the carcinogens in their bodies shows differences. In particular, the activity deficiencies in critical enzymes involved in the detoxification of carcinogens are believed to leading the development of cancer. ${ }^{16}$ In an analysis which is realized by Loktionov et al. ${ }^{17}$ GSTM1, and GSTM3 polymorphisms were reported to be related with the risk of colorectal cancer. ${ }^{17}$ In a different study which is conducted by Aghajany-Nasab et al. ${ }^{18}$ on 230 subjects, they reported that the GSTM1 null genotype was associated with predisposition to the development of colorectal cancer in individuals who were older than 60 years. ${ }^{18}$ Zhao et al. ${ }^{19}$ reported that GSTP1, GSTT1, and GSTM1 gene polymorphisms were not a risk factor for colorectal adenoma in the The Chinese meta-analysis study. ${ }^{19}$

\section{Lung cancer}

This cancer is currently one of the most common types of cancer, and is considered to be one of the leading causes of cancer-related deaths worldwide. ${ }^{20}$ The relationship between the polymorphic 
expression of different GST types, and the risk of lung cancer was investigated in the studies conducted in different ethnic populations, and the results were found to be contradictory. ${ }^{21}$ It is predicted that there may be an important relationship between lung cancer risk, and GSTM1 null genotype in different populations. ${ }^{22}$ In some studies, GSTM1 null genotypes have been reported to play a protective role for cancer. ${ }^{23}$

\section{Oral cancer}

GSTP1 has been reported to be the most prominent GST isoenzyme expressed in oral tissues, and it has been found to be significantly an increased in leukoplakias with premalign or malignant oral lesion, laryngeal tumor, moderate-severe dysplasia, and submucous fibrosis. In the subjects with $A / G$ or $\mathrm{G} / \mathrm{G}$ alleles, there are studies showing that they are included in the higher risk group for oral cancer. ${ }^{24}$ Especially in oral tumors, it is predicted that GSTP1 may contribute to detoxification by way of overexpression. In addition, it has been reported that individuals who carries the allele group of $A / G$ or G/G for GSTP1 may also prevent the excretion of carcinogens by a decrease in enzyme activity. ${ }^{24}$

\section{Ovarian cancer}

In a different analyses about GSTM1 null, GSTT1 null, and GSTP1 Ile/Ile genotypes, especially in combination, GSTs showed a synergistic effect on the development of ovarian cancer. ${ }^{25}$ In a different study demonstrated by Gates et al. reported that the GSTT1 null genotype was related with oral cancer in a particular group of women using genital talc. ${ }^{26}$

\section{Prostate cancer}

Prostate cancer is defined as a malignant disease of the prostate, and is usually seen in men who were older than 50 age, and usually needs a long period of time for improvement to clinical symptoms. ${ }^{27}$ In a Japanese population, a GSTM1 null genotype was reported to elevate the risk of prostate cancer. ${ }^{28}$ In Austria, Germany, and the United States, there weren't found any connection between the risk of cancer and GSTM1 null genotype..$^{29-31}$

\section{Conclusion}

GSTs are a member of the phase II detoxification enzyme family. This detoxification capability is particularly important in cellular protection against environmental and oxidative stress. GST family consists of multiple isozymes associated with functional polymorphic variations. Current studies have been reported that the abnormal expression of GST isozymes has been associated with the progression and development of many different cancer types. This review discusses how changes in the expression profiles of human GST isozymes affect cancer susceptibility. And also, in this review shows that the GSTM1/GSTT1/GSTP1 genotypes in specific cancer types. Our data, therefore, also indicate a significant function on GST enzymes in the detoxification of various carcinogenic molecules which involves the progression of cancers. According to these results, oxidative stress, and depletion of glutathione may act a significant role in the pathogenesis of certain cancer types. ${ }^{32}$

The deletion of GSTM1 and GSTT1 genes is expressed as a "null" genotype, which represents a general lack of enzymatic activity. It is thought that the risk of malignancy is increased as a result of the decrease in the detoxification ability of the possible carcinogens of individuals who are homozygous for these deletions. Where this phenotype was studied as a predictor of cancer prognosis or response to therapy, the results were reported to depend on the type of tumor. In order to reach a clear result, different studies including both a high, and low-risk genotypes with a high number of subjects.

Due to the small scale population studies carried out so far about cancer patients, limited information about this subject has been obtained. Although there are many correlative reports on this subject, it is very difficult to obtain clear results from these data. What we can clearly say is that it is unlikely that a single variable represented only by GST polymorphism can be considered as a simple risk factor for detection and analysis of cancer sensitivity. Many other variables such as exposure to carcinogens, genetic polymorphisms and population diversity should be taken into account in the context of the predisposition mechanism. When the current literature review is performed, it is seen that there are many epidemiological reports related to the connection between GST expression and cancer incidence in small sample size. GST, a phase II detoxification enzyme, is not likely to act alone in the predisposition to cancer. For this reason, polymorphism studies including large-scale and different populations of other phase I and phase II detoxification enzymes are investigated. However, in such a way, it can be expressed more clearly whether GST expression profiles contribute to disease progression and prognosis. ${ }^{32}$

\section{Acknowledgements}

None.

\section{Conflict of interest}

Author declares that there is no conflicts of interest.

\section{References}

1. Seidegard J, Vorachek WR, Pero RW, et al. Hereditary differences in the expression of human glutathione transferase active on trans-stilbene oxide are due to a gene deletion. Proc Natl Acad Sci USA. 1988; 85(19):72937297.

2. Yang X, Qiu MT, Hu JW, et al. GSTT1 null genotype contributes to lung cancer risk in asian populations: a meta-analysis of 23 studies. PLoS One. 2013; 8:e62181.

3. Zhao Y, Luo Y, Huang B, et al. GSTT1 null genotype contributes to an increased risk of gastric cancer in the chinese population: evidence from a meta-analysis. Tumour Bio. 2013;34:1691-1697.

4. Vineis P. Epidemiology of cancer from exposure to arylamines. Environ Health Perspect. 1994;102:7-10.

5. Simic T, Savic RA, Pljesa EM, et al. Glutathione S-transferases in kidney and urinary bladder tumors. Nat Rev Urol. 2009;6: 281-289.

6. Lee SJ, Cho SH, Park SK et al. Combined effect of glutathione S-transferase M1 and T1 genotypes on bladder cancer risk. Cancer Lett. 2002;177:173-179.

7. Kim WJ, Lee HL, Lee SC, et al. Polymorphisms of N-acetyltransferase 2 , glutathione S-transferase mu and theta genes as risk factors of bladder cancer in relation to asthma and tuberculosis. J Urol. 2000;164:209-213.

8. Sosa V, Moliné T, Somoza R, et al. Oxidative stress and cancer: an overview. Ageing Res Rev. 2013;12(1):376-90.

9. Yager JD, Davidson NE. Estrogen carcinogensesis in breast Cancer. $N$ Engl J Med. 2006;354:270-282. 
10. Sá RA, Moreira AD, Cabello PH, et al. Human glutathione S-transferase polymorphisms associated with prostate cancer in the Brazilian population. International braz j urol. 2014;40(4):463-473.

11. Fang J, Wang S, Zhang S, et al. Asociation of the glutathione s-transferase m1, t1 polymorphisms with cancer: evidence from a meta-analysis. PLoS ONE. 2013;8:78707.

12. Possuelo LG, Peraça CF, Eisenhardt MF, et al. Polymorphisms of GSTM1 , and GSTT1 genes in breast cancer susceptibility: a case-control study. Rev Bras Ginecol Obstet. 2013;35:569-574

13. Samson M, Swaminathan R, Rama R, et al. Asian pacific. J Cancer Prev. 2007;8:253-257.

14. Stigliano V, Sanchez ML, Martayan A, et al. Early-onset colorectal cancer: a sporadic or inherited disease?. World J Gastroenterol. 2014;20:12420 12430 .

15. Brody H. Lung cancer. Nature. 2014;513(7517):S1.

16. Economopoulos KP, Sergentanis TN. GSTM1, GSTT1, GSTP1, GSTA1, and colorectal cancer risk: a comprehensive meta-analysis. Eur J Cancer. 2010;46:1617-1631.

17. Loktionov A. Glutathione-S-transferase gene polymorphisms in colorectal cancer patients: interaction between GSTM1 and GSTM3 allele variants as a risk-modulating factor. Carcinogensesis. 2001;22:1053-1060.

18. Aghajany NM. Glutathione S-transferase mu gene variants, and colorectal cancer development-use of sequence-specific probes for an Iranian population. Asian Pac J Cancer Prev. 2011;12:1511-1515.

19. Zhao ZQ. System review, and meta-analysis of the relationships between five metabolic gene polymorphisms and colorectal adenoma risk. Tumor Biol. 2012;33:523-535.

20. Tota JE, Ramanakumar AV, Franco EL. Lung cancer screening: review , and performance comparison under different risk scenarios. Lung 2014;192(1):55-63.

21. Altinisik J, Balta ZB, Aydin G, et al. Investigation of glutathione S-transferase M1, and T1 deletions in lung cancer. Mol Biol Rep. 2010;37(1):263-267.
22. Liu D, Wang F, Wang Q, et al. Association of glutathione S-transferase M1 polymorphisms and lung cancer risk in a The Chinese population. Clin Chim Acta. 2012;414:188-190.

23. Yadav DS, Devi TR, Ihsan R, et al. Polymorphisms of glutathione-Stransferase genes and the risk of aerodigestive tract cancers in the Northeast Indian population. Genet Test Mol Biomarkers. 2010;14(5):715-723.

24. Mulder TP, Manni JJ, Roelofs HM, et al. Glutathione S-transferases, and glutathione in human head and neck cancer. Carcinogensesis. 1995;16(3):619-24.

25. Lallas TA, Mcclain SK, Shahin MS, et al. The glutathione S-transferase M1 genotype in ovarian cancer. Cancer Epidemiol Biomarkers Prev. 2000;9:587-590.

26. Gates MA, Tworoger SS, Terry KL, et al. Talc use, variants of the GSTM1, GSTT1, , and NAT2 genes and risk of epithelial ovarian cancer. Cancer Epidemiol Biomarkers Prev. 2008;17:2436-2444.

27. Siegel R, Ward E, Brawley O, et al. Cancer statistics, 2011: the impact of eliminating socioeconomic and racial disparities on premature cancer deaths. CA Cancer J Clin. 2011;61(4):212-236.

28. Murata M, Shiraishi T, Fukutome K, et al. Cytochrome P4501A1, and glutathione S-transferase M1 genotypes as risk factors for prostate cancer in Japan. Jpn J Clin Oncol. 1998;28:657-660.

29. Gsur A, Haidinger G, Hinteregger S, et al. Polymorphisms of glutathioneS-transferase genes (GSTP1, GSTM1 and GSTT1) and prostate cancer risk. Int J Cancer. 2001;95:152-155.

30. Steinhoff C, Franke KH, Golka K, et al. Glutathione transferase isozyme genotypes in patients with prostate and bladder carcinoma. Arch Toxicol. 2000;74:521-526.

31. Rebbeck TR, Walker AH, Jaffe JM, et al. Glutathione S-transferase-mu (GSTM1) and - theta (GSTT1) genotypes in the etiology of prostate cancer. Cancer Epidemiol Biomarkers Prev. 1999;8:283-287.

32. McIlwain CC, Townsend DM, Tew KD. Glutathione S-transferase polymorphisms: cancer incidence and therapy. Oncogene. 2006;25(11):1639-1648. 